\title{
NOISE AND DRIFT CHARACTERIZATION OF DIRECT LASER TO RF CONVERSION SCHEME FOR THE LASER BASED SYNCHRONIZATION SYSTEM FOR FLASH AT DESY
}

\author{
B. Lorbeer, F. Ludwig, H. Schlarb, A. Winter, DESY, Hamburg, Germany
}

\section{Abstract}

Next generation FELs (Free Electron Lasers) require a long and short term stable synchronization of RF reference signals with an accuracy of $10 \mathrm{fs}$. To overcome the limitations of a coaxial cable based system, an optical synchronization system is being developed for FLASH at DESY. It is based on the distribution of sub-ps optical pulses, where the timing information is encoded in the precise repetition rate. The optical pulse train has to be converted into an RF signal to provide a local reference for calibration and operation of RF based devices. The drift and jitter performance of the optical to RF converter influences directly the phase stability of the accelerator. Three different methods for optical to RF converters, namely the direct detection using a photodiode, injection locking of a DRO, and a sagnac-loop interferometer are currently under investigation. In this paper we concentrate on the jitter and drift performance of the direct photodiode conversion and show its limitations.

\section{INTRODUCTION}

An optical synchronization system based on the generation and distribution of sub-ps laser pulses [1] is currently under development for FLASH at DESY to overcome the short and long term stability limitations of a conventional, coax based RF distribution system [2]. The tight stability requirements for the laser master oscillator (LMO) can be met by phase locking a passively mode locked Erbium doped fiber laser [4], which in itself has excellent highfrequency noise properties [5], to a microwave oscillator for low-frequency stabilization. The optical pulse train is distributed via length stabilized fiber links to various locations within the facility [3]. The length stabilization is done using optical cross-correlation, where part of the pulse is reflected back at the end of the fiber link and combined with a pulse directly from the laser. The arrival time changes are corrected using a piezo-based fiber stretcher [3]. A short and long-term stable RF signal at $1.3 \mathrm{GHz}$ needs to be generated from the optical pulse train at the end of a link. Three different methods have previously been shown to be a candidate for this purpose, namely direct photodiode detection, injection locking of a separate oscillator, and a sagnac loop interferometer [6]. Besides investigating the phase noise performance of the direct photodiode detection, we present a miniaturized and improved temperature stabilization setup to overcome phase drifts of the reconverted RF signal due to temperature changes in the detection chain.

\section{DIRECT CONVERSION SCHEME}

The optical pulse train from an EDFL is fed to a photodiode that generates an electrical pulse train with a repetition rate $f_{\text {rep }}=54 \mathrm{MHz}$ given by the laser. The electrical pulses after photo detection consist of fourier components up to the photodetector bandwidth, equally spaced at the repetition rate of the pulses. We extract the $24^{\text {th }}$ harmonic of the pulse train at $1.3 \mathrm{GHz}$ with a bandpass filter and amplify the signal using a low noise amplifier with a gain of approximately $G=30 \mathrm{~dB}$. The measurement principle is sketched in figure 1.

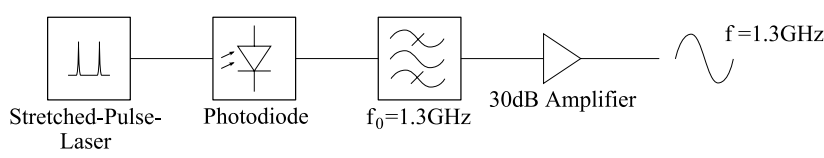

Figure 1: Direct photodiode conversion scheme for extracting $1.3 \mathrm{GHz}$. The components are: a $10 \mathrm{GHz}$ photodiode from EOTECH, a bandpass filter from IMCSD and a low noise amplifier ZRL-1150LN from Minicircuits.

\section{Phase Noise}

The short term stability of the direct conversion scheme is studied in terms of phase noise. We measure the single sideband phase noise in a bandwidth from $f_{1}=1 \mathrm{kHz}$ to $f_{2}=20 \mathrm{MHz}$ and obtain the integrated timing jitter by

$$
\Delta T_{\mathrm{rms}}=\frac{1}{2 \pi f_{0}} \sqrt{\int_{f_{1}}^{f_{2}} S_{\phi}(f) d f}
$$

where $S_{\phi}(f)$ is twice the measured single sideband phase noise $\mathscr{L}(f)$ [8]. The phase noise spectra are shown in figure 2.

Compared to the phase noise performance of measurements that have been reported recently ${ }^{1}$ [5] we have two times worse integrated timing jitter in our conversion chain. For this measurement the laser was not optimized in phase noise for the extracted RF signal. A phase noise characteristic where the laser was optimized for phase noise performance is added in the graph. The best phase noise performance can be achieved by minimizing the amplitude fluctuations of the optical pulse train since the photodiodes might have a high AM to PM conversion coefficient.

\footnotetext{
${ }^{1}$ we measured 9 fs integrated timing jitter in the same bandwidth
}

T23 Timing and Synchronization 


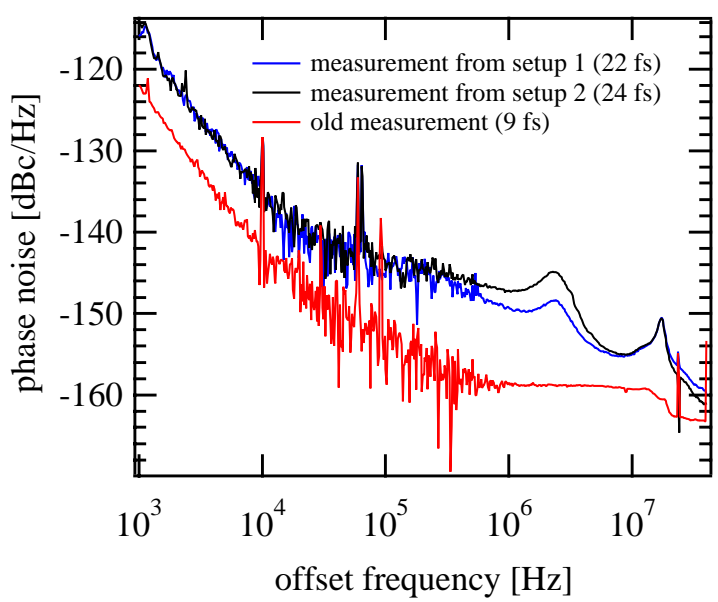

Figure 2: Phase noise of the $24^{\text {th }}$ harmonic of the laser repetition rate, measured by an Agilent SSA 5052A. Integration yields a timing jitter of $24 \mathrm{fs}, 22 \mathrm{fs}$ and 9 fs respectively in a bandwidth from $1 \mathrm{kHz}$ to $20 \mathrm{MHz}$.

\section{Long Term Drifts}

We obtain the long term stability of the direct conversion scheme by comparing the extracted RF phases of two separate laser to RF converters in a phasedetector and record the voltage fluctuations over a timescale of hours. The setup is depicted in figure 3. The recorded voltage fluctuations $\Delta V$

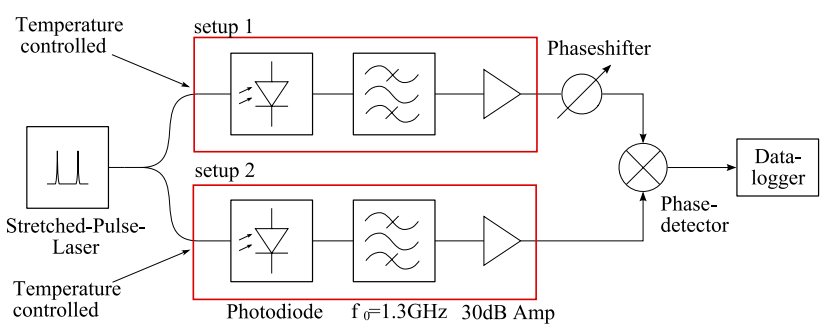

Figure 3: Drift measurement setup with two independent temperature controls for setup 1 and 2 .

are converted to phase fluctuations $\Delta \phi$ using the conversion gain $K_{\phi}$ of the phase detector

$$
\Delta \phi=\frac{\Delta V}{K_{\phi}}
$$

and then related to the period $T_{0}$ of the carrier with frequency $f_{0}=1.3 \mathrm{GHz}$

$$
\Delta T=\frac{\Delta \phi}{360^{\circ}} T_{0} .
$$

Temperature stabilization has been improved by constructing a miniaturized setup (4) of the converter components (photodiode, bandpass and amplifier) to minimize the local temperature dependent drifts according.

The measurement limitations of the setup when both converters are kept at a constant temperature $\left(<0.05^{\circ} \mathrm{C}\right.$ peak-peak ) is shown in figure 5 . In this measurement we 07 Accelerator Technology Main Systems

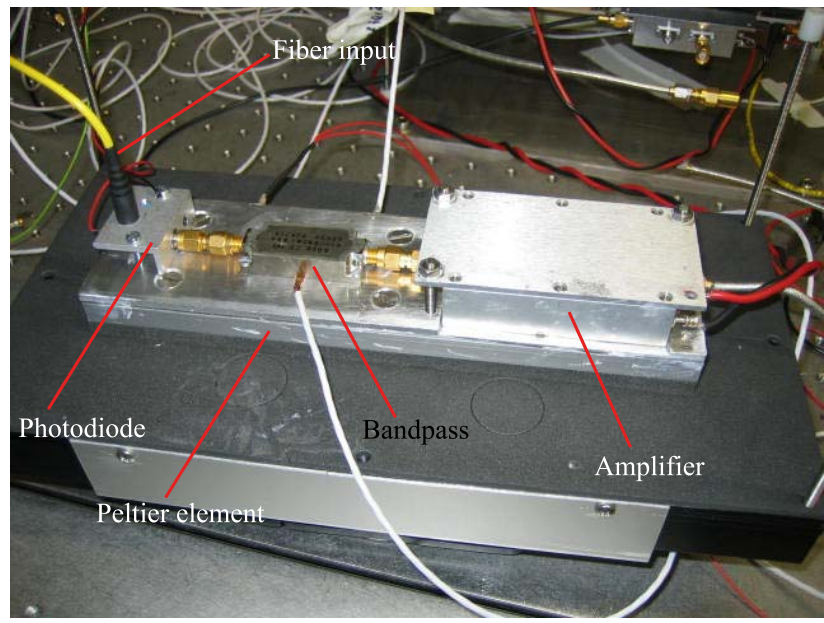

Figure 4: Miniaturized setup 1 with peltier temperature control.

observe phase drifts of about $80 \mathrm{fs}$ (peak-peak) ( $35 \mathrm{fs}_{\text {rms }}$ ) over a time of 4 hours and measured with a bandwidth of $1 \mathrm{kHz}$. The limitations of the phasedetector is better than $50 \mathrm{fs}$ (peak-peak) ( $8 \mathrm{fs}_{\mathrm{rms}}$ ) over a time period of 4 hours [5] which is a conservative number but reproducible. The best measurement results for a phase detector measurement excluding the photodiodes and instead making use of a splitter and an RF source is about 20 fs (peak-peak). When applying temperature steps to one of the converters as shown in figure 6 and keeping the other one as well as the phasedetector at a constant temperature (better than $0.05^{\circ} \mathrm{C}$ ), it is possible to extract the temperature coefficient for different temperature steps and operation temperatures.

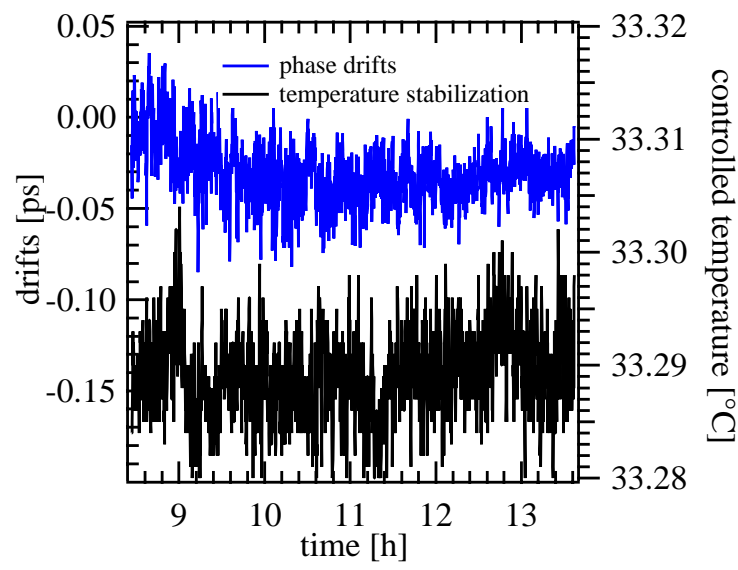

Figure 5: Measurement limitation of direct conversion scheme.

The temperature coefficients are summarized in table 1.

From the temperature coefficients one may conclude that a temperature stability of $0.05^{\circ} \mathrm{C}$ (peak-peak) like in our measurement results in a phase stability of $18.3 \mathrm{fs}$. This contradicts our measured 80 fs obviously caused by other 


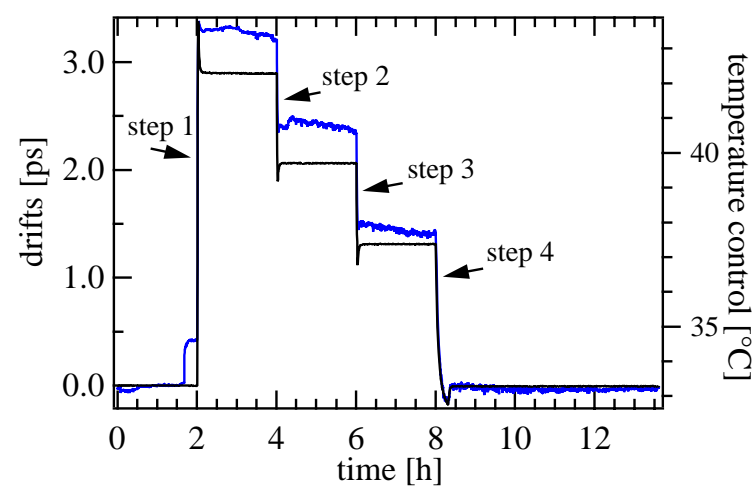

Figure 6: Temperature steps on laser to RF converter: blue trace are drifts, black trace is temperature of setup.

Table 1: Temperature coefficients of direct conversion scheme.

\begin{tabular}{|l|c|}
\hline Step number & temperature coefficient $\left[\mathbf{f s} /{ }^{\circ} \mathbf{C}\right]$ \\
\hline Step 1 & 366 \\
Step 2 & 326 \\
Step 3 & 359 \\
Step 4 & 350 \\
\hline
\end{tabular}

noise sources. We exclude the length variation of the fibers that connect the laser source with the photodiodes.

The origin of phase drifts might be caused by different $\mathrm{AM}$ to PM conversion coefficient of the converters. The AM to PM conversion coefficients for different photodiodes ranges between $500 \mathrm{fs} / \mathrm{mW}$ to $4 \mathrm{ps} / \mathrm{mW}$ [5]. In this context the amplitude fluctuations of the non optimized laser will lead to different phase changes of the electrical pulse train. An optical amplitude stabilization will reduce the AM to PM of the photodiodes.

\section{CONCLUSION AND OUTLOOK}

We measured residual phase drifts in the direct laser to RF conversion chain involving a photodiode, a $1.3 \mathrm{GHz}$ bandpass filter, and a low noise amplifier of $80 \mathrm{fs}$ (peakpeak)( $\left.35 \mathrm{fs}_{\mathrm{rms}}\right)$. Compared to earlier measurements, the RF phase drifts could not be reduced by improving the temperature stabilization of the setup. The temperature coefficient is in the order of $330 \mathrm{fs} /{ }^{\circ} \mathrm{C}$, meaning the measured drift is four times higher than expected. Therefore, there must be another source for these drifts.The different phase noise characteristics of our detection chain presented in this paper compared to our earlier published results [5] is explainable from a higher AM to PM conversion contribution that varies from photodiode to photodiode and a non optimized tuning of the laser. In addition, the long term stability of the phase detectors has to be improved by applying self calibration or bridge techniques [7].

Injection locking of an oscillator using a photodiode and an oscillator with integrated timing jitter smaller than $4 \mathrm{fs}$ in the frequency range from $1 \mathrm{kHz}$ to $20 \mathrm{MHz}$ [9] cleans up the phase noise of the $1.3 \mathrm{GHz}$ spectral component of the pulse repetition rate. At least for laser repetition rates of $54 \mathrm{MHz}$ the phase noise of injection locking is expected to be smaller than for direct conversion. Phase drifts of both methods are expected to be comparable and limited by AM to PM noise from the photodiode, which has to be investigated. The phase drift and phase noise limitation of both mentioned methods might be overcome by a balanced phase detection scheme proposed in [6]. We acknowledge financial support by the EUROFEL project.

\section{REFERENCES}

[1] J. Kim et. al., "An integrated femtosecond timing distribution system for XFEL's”, EPAC 2006, Edinburgh, UK, 2006

[2] K. Czuba et. al., "Master Oscillator Design For The VUV FEL Project", MIKON conference 2006

[3] F. Loehl et. al., "First prototype of an optical cross-correlation based fiber-link stabilization for the FLASH synchronization system,” Dipac 2007, Venice, Italy, 2007

[4] A. Winter et. al., "High-precision laser master oscillators for optical timing distribution systems in future light sources", EPAC 2006, Edinburgh, UK, 2006

[5] B. Lorbeer et. al., "Noise and drift characterization of critical components for the laser based synchronization system at FLASH”, Dipac 2007, Venice, Italy, 2007

[6] J. Kim et. al., "Balanced optical-microwave phase detectors for optoelectronic phase-locked loops", Opt. Lett. 31, 3659(2006)

[7] J. Mueller, "Selbstkalibrierender Phasendetektor", Desy Thesis, Studienarbeit 2006, DESY, Hamburg, Germany

[8] M. Thumm et. al., "Hochfrequenzmesstechnik. Verfahren und Meßsysteme”, B.G. Teubner Stuttgart, September 1998 ISBN 3519163608

[9] P. Stockwell et. al., "A low phase noise $1.3 \mathrm{GHz}$ dielectric resonator oscillator",Poseidon Scientific Instruments, Fremantle, Australia 